\title{
Supporting Information: Transpiration Mechanism in Confined Nanopores
}

\author{
An Zou, Manish Gupta, and Shalabh C. Maroo* \\ Department of Mechanical and Aerospace Engineering, Syracuse University, Syracuse, \\ NY 13244 \\ *Corresponding Author: scmaroo@syr.edu
}

\section{S1 Molecular Dynamics Simulations}

\section{Simulation Domains}

We created two separate simulation domains (Fig. 1a and Fig. 4a in the main manuscript). The first domain, which is for simulation validation and study of transition from positive to negative liquid pressure, is symmetric in $y$ direction and the hydrophilic surface (HL) at each end consists of five-layer FCC $<111>$ plane atoms. A certain number of layers of Ar atoms with density of $\sim 1400 \mathrm{~kg} / \mathrm{m}^{3}$ were placed in the domain according to the desired film thickness, 11 layers of atoms with density of $\sim 7.4 \mathrm{~kg} / \mathrm{m}^{3}$ were placed at the center of the domain as vapor. The second domain, which is to simulate evaporation in nanopores, included a $5 \mathrm{~nm}$ nanopore formed between two hydrophilic surfaces, which are the same as in first domain. A $5.2 \mathrm{~nm}$ thick continuous film served as bulk liquid. A $1.2 \mathrm{~nm}$ thick hydrophobic (HP) surface was added to the left edge of domain to prevent the direct interaction between bulk liquid and vapor, which is present at the other side of the domain. The space between the hydrophilic surface and the simulation domain boundary in $x$ direction was filled with the same hydrophobic atoms.

\section{Atomic Interactions}

In all simulations, the atomic interaction among Ar atoms is governed by standard Lennard - Jones (L-J) potential $\left(\phi_{L J}=4 \varepsilon\left[\left(\frac{\sigma}{r}\right)^{12}-\left(\frac{\sigma}{r}\right)^{6}\right]\right.$, where $\varepsilon$ is the depth of the potential well, $\sigma$ is the distance where the potential is zero, and $r$ is the distance between two atoms) with $\varepsilon_{A r-A r}=1.67 \times 10^{-21} \mathrm{~J}$ and $\sigma_{A r-A r}=3.400 \times 10^{-10} \mathrm{~m}$. The cut-off distance $\left(r_{c}\right.$ for Ar-Ar interaction was set as $1.2 \mathrm{~nm}(\sim 3.5 \sigma)$ for which the bulk liquid equilibrium density was $\sim 1350$ $\mathrm{kg} / \mathrm{m}^{3}$ at $90 \mathrm{~K}$. The lower cut-off of $1.2 \mathrm{~nm}$ was used in current work to save computation cost. The hydrophilic surface is mimicked by using modified L-J potential [1] to represent atomic interaction between a single surface atom (HL) and Ar with high $\varepsilon$ value of $2.49 \times 10^{-21}$ $\mathrm{J}$ and $\sigma$ of $3.085 \times 10^{-10} \mathrm{~m}$ in Eq. S1. To eliminate the effect of hydrophobic surface on liquid properties, its interaction with argon atoms was governed by standard L-J potential with $\varepsilon_{A r-H B}=0.01 \varepsilon_{A r-A r}=1.67 \times 10^{-23} \mathrm{~J}$ and $\sigma_{A r-B}=3.085 \times 10^{-10} \mathrm{~m}$. Table S1 summarizes the parameters used for atomic interactions for all combinations.

$$
\begin{aligned}
\phi_{A r-H L}= & 4 \varepsilon\left[\left(\frac{\sigma}{r}\right)^{9}-\left(\frac{\sigma}{r}\right)^{5}\right]+\left(1-\frac{r_{c}}{r}\right) 4 \varepsilon\left[9\left(\frac{\sigma}{r_{c}}\right)^{9}-5\left(\frac{\sigma}{r_{c}}\right)^{5}\right] \\
& -4 \varepsilon\left[\left(\frac{\sigma}{r_{c}}\right)^{9}-\left(\frac{\sigma}{r_{c}}\right)^{5}\right]
\end{aligned}
$$

where $r_{c}$ is the cut-off distance. 
Table S1: L-J Potential Parameters

\begin{tabular}{ccccc}
\hline Combination & Type & $\varepsilon\left(10^{-21} \mathrm{~J}\right)$ & $\sigma(\mathrm{nm})$ & $r_{c}(\mathrm{~nm})$ \\
\hline $\mathrm{Ar}-\mathrm{Ar}$ & $12-6$ & 1.67 & 0.3400 & 1.2 \\
$\mathrm{Ar}-\mathrm{HL}$ & $9-5$ & 2.49 & 0.3085 & 2.0 \\
$\mathrm{Ar}-\mathrm{HP}$ & $12-6$ & 0.0167 & 0.3085 & 1.2 \\
\hline
\end{tabular}

\section{S2 Liquid Film on Hydrophilic Surface}

\section{Density/Pressure Profiles}

To obtain the local density and pressure in the film, the domain was divided into a certain number of bins with constant size. The number of atoms in each bin was used to calculate the local density (Eq. S2); while the normal stresses for each atom in current bin was summed up to obtain the local pressure (Eq. S3) [2].

$$
\begin{gathered}
\rho_{i}=\frac{N_{i} M_{A r}}{N_{A} V_{i}} \\
P_{i}=-\frac{1}{3 V_{i}} \sum_{k=x, y, z}\left[\frac{M_{A r}}{N_{A}} v_{k}^{2}-\frac{1}{2} \sum_{n=1}^{N_{p}} \frac{d_{k}}{r} \phi^{\prime}(r)\right]
\end{gathered}
$$

where $N_{i}$ is number of atoms in $i$ th bin, $M_{A r}$ is molecular mass of Ar, $N_{A}$ is Avogadro number, $V_{i}$ is volume of the bin, $v_{k}$ is the atom velocity at $k$ direction. The first term in summation of Eq. S3 is the kinetic energy contribution; while the second term is pairwise energy contribution where $n$ loops over the $N_{p}$ neighbors of atom, $d_{k}$ is the distance between two atoms in $k$ direction, and $\phi^{\prime}(r)$ is the first order derivative of L-J potential with respect to $r$. Constant $1 / 2$ means that if only one atom of the pair is in $i$ th bin, half of the intermolecular force contribution is given to the current bin; total contribution is given to current bin if both atoms locate in the same bin.

8 cases were run with film thickness varying from $0.65 \mathrm{~nm}$ to $15.76 \mathrm{~nm}$. Fig. S1 shows density and pressure profiles in the film at the equilibrium state as function of the distance to surface, $d$, in all cases. The profiles were obtained with two different bin sizes, $0.05 \mathrm{~nm}$ and 0.30 $\mathrm{nm}$. Extreme high local density and pressure were observed close to surface using $0.05 \mathrm{~nm}$ bins. The fluctuation of both density and pressure corresponds to the layered liquid atoms, which was not captured using $0.30 \mathrm{~nm}$ bins.

The high pressure in surface-affected region was observed as long as the film this "thick" enough to fully fill the layers where the liquid atoms were aligned as FCC $<111>$ plane, which is adopted from the neighboring surface atoms (Fig. S2-a); otherwise, negative pressure in the film was observed, such as the film with thickness of $0.65 \mathrm{~nm}$ (Fig. S2-b). 

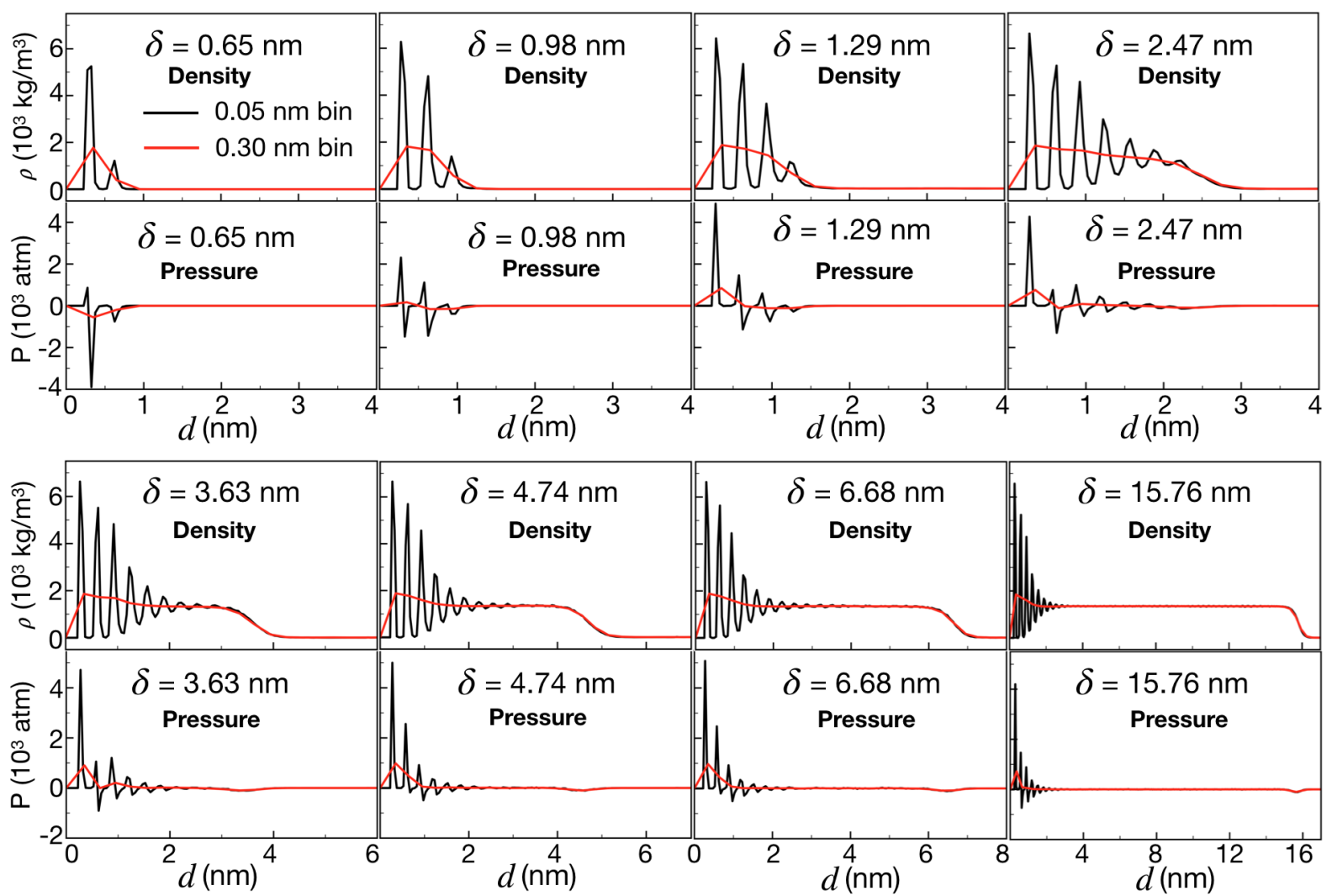

Figure S1: Density and pressure profiles as function of distance to surface $(d)$ for all cases with different bin sizes: $0.05 \mathrm{~nm}(\longrightarrow)$ and $0.30 \mathrm{~nm}(\square)$.

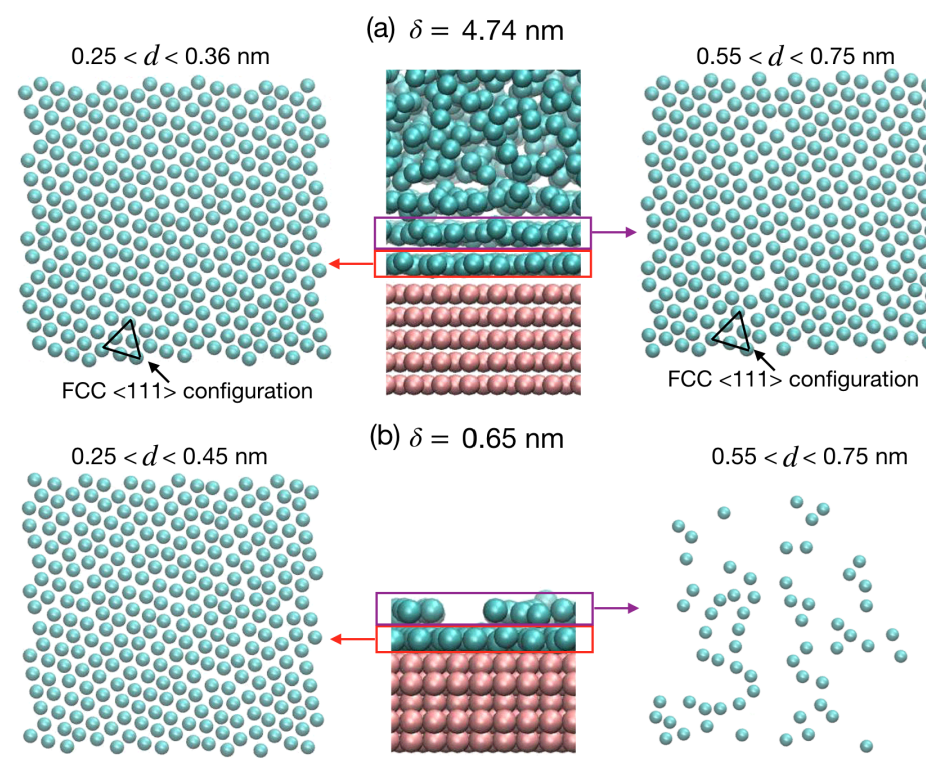

Figure S2: Layered liquid atoms in the film.

\section{Equilibrium Vapor Pressure}

The equilibrium vapor pressure of a film was predicted by classic disjoining pressure theory, which had been reported to be valid for cases similar to our simulations [3, 4]. The derivation of equilibrium vapor pressure of a thin film with presence of disjoining pressure effects was adopted 


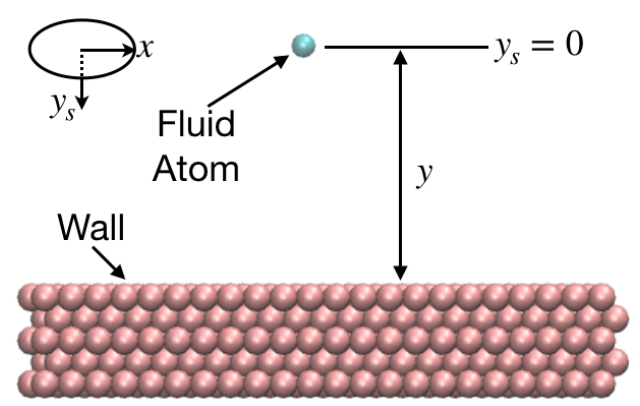

Figure S3: System for classic thermodynamic and hydrodynamic analysis.

from literature $[3,5]$, which starts from Gibbs-Duhem equation (Eq. S4).

$$
d \hat{\mu}=-\hat{s} d T+\hat{v} d P
$$

where $\hat{\mu}$ is molar chemical potential; $\hat{s}$ is molar entropy; $T$ is temperature; $\hat{v}$ is molar specific volume; and $P$ is pressure. Integrating Eq. S4 at constant temperature from saturation temperature to an arbitrary state in liquid and vapor phases respectively by treating liquid as incompressible and vapor as an ideal gas. By doing so, The chemical potentials of liquid and vapor without surface attraction effect can be obtained as Eqs. S5 and S6.

$$
\begin{gathered}
\hat{\mu}_{v}=\hat{\mu}_{v, s a t}+\bar{R} T \ln \left(P_{v e} / P_{s a t}\right) \\
\hat{\mu}_{l}=\hat{\mu}_{l, s a t}+\hat{v}_{l}\left(P_{l}-P_{s a t}\right)
\end{gathered}
$$

With surface attraction effect in a film of pure liquid, the chemical potential is augmented by the potential energy due to the intermolecular interactions between surface atoms and liquid atoms. Thus, Eq. S7 can be obtained.

$$
\hat{\mu}_{l}=\hat{\mu}_{l, s a t}+\hat{v}_{l}\left(P_{l}-P_{s a t}\right)+N_{A} \phi_{s}
$$

where $N_{A}$ is Avogadro number; $\phi_{w}$ is the potential energy felt by a single free fluid atom due to the interaction with the entire surface.

At equilibrium, the chemical potentials of liquid phase and vapor phase must be the same. Thus, Eq. S8 can be obtained by combining Eqs. S6 and S7.

$$
\bar{R} T \ln \left(P_{v e} / P_{\text {sat }}\right)=\hat{v}_{l}\left(P_{l}-P_{\text {sat }}\right)+N_{A} \phi_{s}
$$

Eq. S8 can be rewritten as

$$
\frac{P_{v e}}{P_{s a t}}=\exp \left\{\frac{P_{s a t} \hat{v}_{l}}{\bar{R} T}\left(\frac{P_{l}}{P_{s a t}}-1\right)+\frac{N_{A} \phi_{s}}{\bar{R} T}\right\}
$$

In our simulations, the pressure in bulk liquid film is close to saturation pressure, causing the first term in the curly brackets in Eq. S9 to be negligible. Thus, Eq. S10 can be obtained, in which only $\phi_{w}$ is unknown.

$$
\frac{P_{v e}}{P_{\text {sat }}}=\exp \left\{\frac{\phi_{s}}{k_{B} T}\right\}
$$

To obtain the surface potential for a single fluid atom, the atomic interaction between a single surface atom and Ar was simplified as Eq. S11 from Eq. S1, and a system consists of a single fluid atom and an infinity thick surface as shown in Fig. S3. 


$$
\phi_{A r-H L}=4 \varepsilon\left[\left(\frac{\sigma}{r}\right)^{9}-\left(\frac{\sigma}{r}\right)^{5}\right]
$$

The surface potential can be written as

$$
\phi_{s}=\int_{y_{s}=y}^{\infty} \int_{x=0}^{\infty} \rho_{s} \cdot \phi_{A r-W A} \cdot(2 \pi x) \cdot d x \cdot d y_{s}
$$

where $\rho_{s}$ is number density of surface atoms. Substituting Eq. S11 and $r^{2}=x^{2}+y^{2}$ into Eq. S12, and integrating yields

$$
\phi_{s}=8 \pi \varepsilon \rho_{s} \sigma^{3}\left[\frac{1}{42}\left(\frac{\sigma}{y}\right)^{6}-\frac{1}{6}\left(\frac{\sigma}{y}\right)^{2}\right]
$$

Defining a constant $C=4 \varepsilon \cdot \sigma^{5}$, then a modified Hamaker constant can be written as

$$
A_{l s}=\pi^{2} \rho_{f} \rho_{s} C
$$

where $\rho_{f}$ is number density of fluid atoms. Substituting Eq. S14 into Eq. S13 yields

$$
\phi_{s}=\frac{A_{l s}}{\pi \rho_{f} \sigma^{2}}\left[\frac{1}{21}\left(\frac{\sigma}{y}\right)^{6}-\frac{1}{3}\left(\frac{\sigma}{y}\right)^{2}\right]
$$

Substituting Eq. S15 to Eq. S10 with ignored $y^{-6}$ term yields Eq. S16, which is used to predict equilibrium vapor pressure of a thin film including disjoining pressure effects.

$$
\frac{P_{v e}}{P_{s a t}}=\exp \left\{-\frac{A_{l s}}{3 \pi \rho_{f} k_{B} T} \cdot \frac{1}{\delta^{2}}\right\}
$$

\section{S3 Pressure Estimation in Nanopore during Liquid Flow}

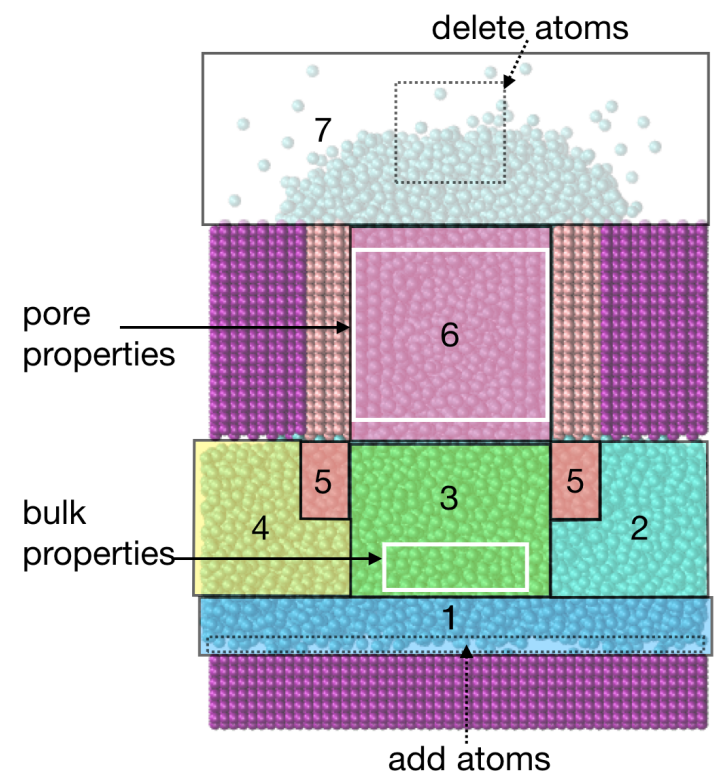

Figure S4: Subregions in simulation domain for pressure calculation. 
Only thermal velocity should be included in the kinetic energy contribution for calculations of pressure and temperature. Thus, to remove the flow velocity induced by evaporation, the simulation domain was divided into a certain number of subregions where the local flow velocity is uniform. To obtain the velocity distribution, the average velocity in each bin $(0.5 \mathrm{~nm} \times$ $0.5 \mathrm{~nm}$ ) was obtained as shown in Fig. 4e in main manuscript, based on which the simulation domain was divided into 7 subregions roughly as shown in Fig. S4, where the white boxes represent the regions for calculating average density/pressure in bulk and pore respectively. An independent thermostat (Nose-Hoover thermostat) was applied in each subregion to maintain local temperature as constant $90 \mathrm{~K}$. The velocity of center of mass in each subregion was calculated as well, and was subtracted for each local atom when calculating pressure.
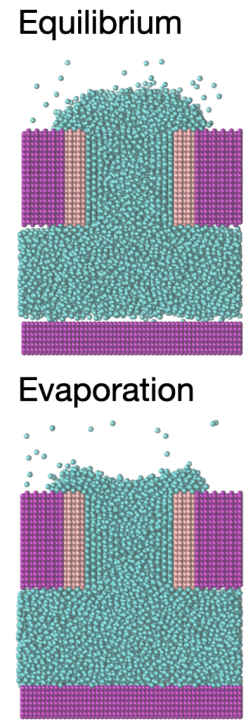
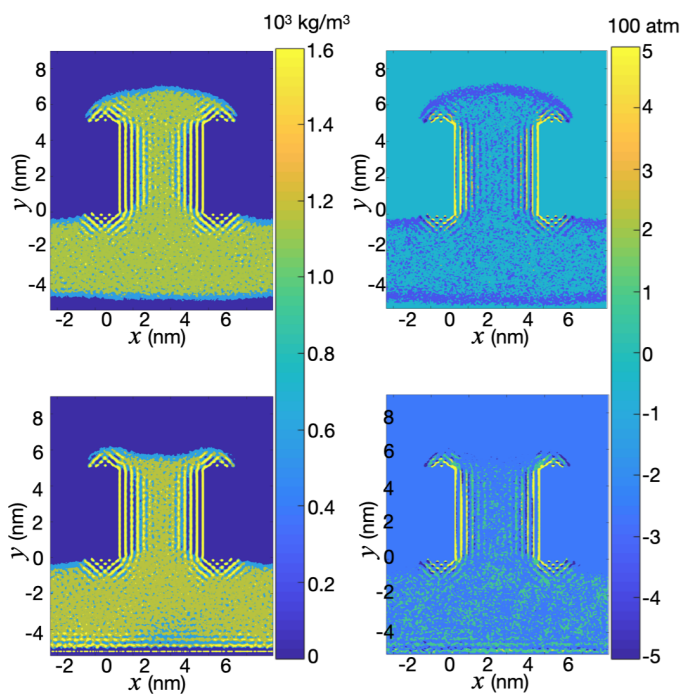

Figure S5: Contour plots of density and pressure for both equilibrium state and steadyevaporation state

Figure S5 shows the 2D distribution of density and pressure in the system for both equilibrium state $(t=2.0 \mathrm{~ns})$ and steady-evaporation state $(t=2.7 \mathrm{~ns})$. Similar to the plots of property profiles across the pore in Figs. $4 \mathrm{c}$ and $4 \mathrm{~d}$ in the main manuscript, density and pressure variations occur near the hydrophilic surface due to the structured liquid layers. The density and pressure distributions in pore during steady-evaporation was largely maintained similar to those in the equilibrium.
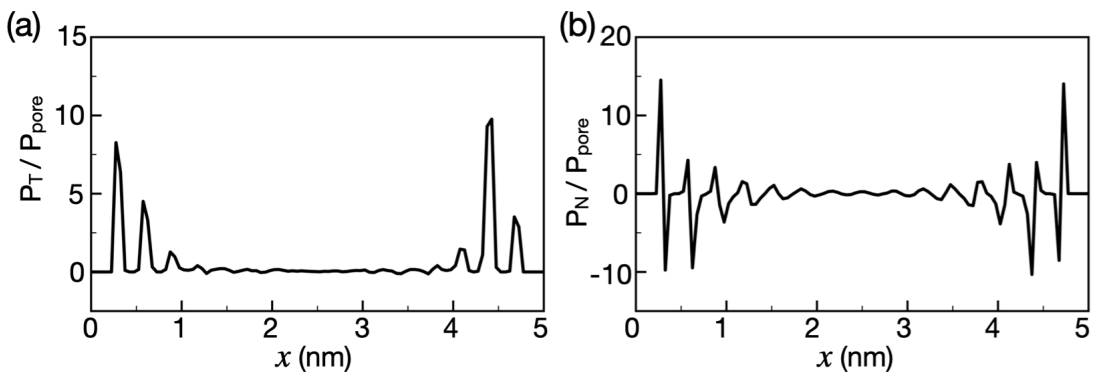

Figure S6: Profiles of ratio of (a) tangential pressure and (b) normal pressure in the pore with respect to the averaged pore pressure during steady-evaporation state.

Figure $\mathrm{S} 6$ shows the distributions of tangential pressure $\left(P_{T}=\frac{1}{2}\left(P_{y y}+P_{z z}\right)\right)$ and normal pressure $\left(P_{N}=P_{x x}\right)$ in the pore during the steady-evaporation state respectively. We non- 
dimensionalized pressure components by dividing them by the average total pressure in pore to highlight the contribution of each component to the total pressure. Such a ratio for average tangential pressure is obtained to be $P_{T, \text { avg }} / P_{\text {pore }}=0.57$ while for average normal pressure ratio is $P_{N, \text { avg }} / P_{\text {pore }}=-0.14$. The main source of high pressure in pore is tangential pressure due to liquid-liquid and liquid-surface interactions, and causes the liquid to flow parallel to the surface along the pore, as shown in Fig. If of the main manuscript. Similarly, such average ratios are also calculated in the bulk region and are found to be: $P_{T, \text { avg }} / P_{\text {bulk }}=0.32$ and $P_{N, \text { avg }} / P_{\text {bulk }}=0.36$. As expected in the bulk region, normal and tangential pressures have similar contribution to the bulk total pressure.

\section{References}

[1] S. D. Stoddard and J. Ford, "Numerical experiments on the stochastic behavior of a lennardjones gas system," Physical Review A, vol. 8, pp. 1504-1512, Sep 1973.

[2] J.-G. Weng, S. Park, J. R. Lukes, and C.-L. Tien, "Molecular dynamics investigation of thickness effect on liquid films," The Journal of Chemical Physics, vol. 113, no. 14, pp. 5917$5923,2000$.

[3] V. P. Carey and A. P. Wemhoff, "Disjoining pressure effects in ultra-thin liquid films in micropassages - comparison of thermodynamic theory with predictions of molecular dynamics simulations," Journal of Heat Transfer, vol. 128, pp. 1276-1284, 032006.

[4] H. Hu and Y. Sun, "Molecular dynamics simulations of disjoining pressure effect in ultrathin water film on a metal surface," Applied Physics Letters, vol. 103, no. 26, p. 263110, 2013.

[5] V. Carey, Liquid Vapor Phase Change Phenomena: An Introduction to the Thermophysics of Vaporization and Condensation Processes in Heat Transfer Equipment, Second Edition. Taylor \& Francis, 2007. 